\title{
PENSATA
}

Submetida 12.05.2014. Aprovada 03.02.2015

Avaliada pelo processo de double blind review. Editor Científico: Luciano Barin Cruz

DOI: http://dx.doi.org/10.1590/So034-759020150310

\section{(RE)APRESENTANDO A TEORIA DA GESTÃO COMPARATIVA}

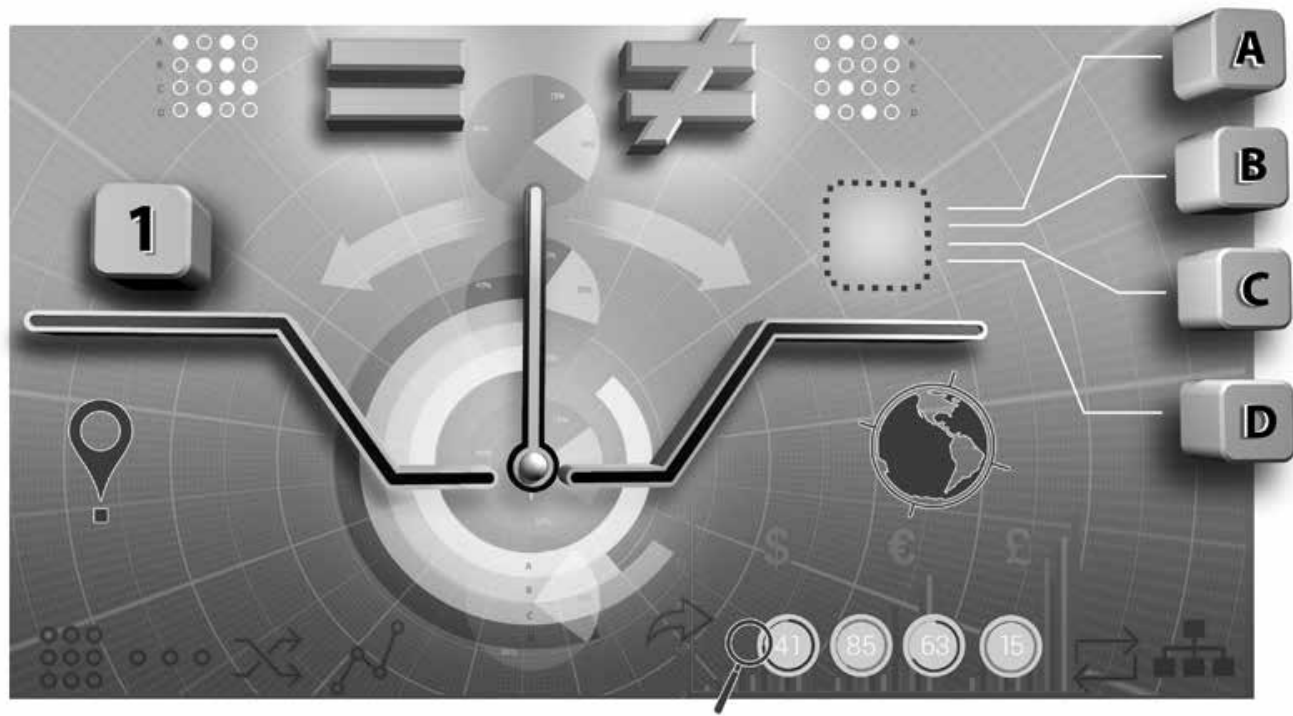

O principal objetivo da teoria da gestão comparativa é prever e explicar a eficiência socioeconômica das organizações que atuam em diferentes países. A partir de contextos nacionais diversos, esse corpo teórico contribui com o desenvolvimento de estudos organizacionais que comparam organizações internacionalizadas quanto aos seus respectivos sistemas de gestão, de produção e de realização de negócios e em relação a organizações instaladas em uma nação de referência. Essa comparação ocorre pela identificação, nos países que amparam essas organizações, das similaridades e das divergências econômicas, culturais, sociais, políticas e institucionais que repercutem nos processos de gestão por elas desempenhados. Por meio desse exercício comparativo, busca-se o aprimoramento gradativo das práticas de internacionalização de empresas (Adler, Doktor, \& Redding, 1986; Alton, 1969; Said, 1978).

As principais revisões sobre o estado da arte da teoria da gestão comparativa são consensuais em relação ao fato de que sua literatura está impregnada por estudos que apenas relatam informa-

\section{RAFAEL BORIM-DE-SOUZA}

borim@uel.br

Professor da Universidade Estadual de Londrina, Centro de Estudos Sociais Aplicados - Londrina - PR, Brasil

\section{ANDRÉA PAULA SEGATTO}

\section{aps@ufpr.br}

Professora da Universidade Federal do Paraná, Centro de Ciências

Sociais e Aplicadas - Curitiba - PR, Brasil ções empíricas e não discutem a implicação teórica desses resultados, o que justifica um desenvolvimento teórico fraco em todos os níveis analíticos. Diversas análises metafóricas sobre esse corpo teórico já foram desenvolvidas, tais como a selva explorada por Schollhammer (1969), o elefante de Roberts (1970), o avestruz de Adler (1983), o dinossauro de Boyacigiller e Adler (1991) e os fósseis buscados por Redding (1994), e todas trouxeram ao meio acadêmico um incômodo pela ausência de maior robustez reflexiva e um interesse de contribuir com uma fundamentação mais densa para a teoria da gestão comparativa.

A maioria das pesquisas desenvolvidas a partir da teoria da gestão comparativa representa um exercício analítico pautado por uma lógica colonialista e, por consequência, afiliado a uma epistemologia positivista. Os autores deste ensaio reconhecem a necessidade de superação dos vieses 
positivistas, mas por um caminho de construção teórica que não incorra nos mesmos erros dessa epistemologia, ou seja, de calar vozes que representem concepções alternativas sobre um mesmo fenômeno. Este ensaio compactua com as reivindicações de alguns autores (Hoogvelt, 2001; Katz, 2001; Kelley \& Worthley, 1981; Kochan, 1983; Peterson, 2001; Pfeffer, 2005; Raghuram \& Madge, 2006; Schollhammer, 1975; Tsui, 2007) ao pretender (re)apresentar a teoria da gestão comparativa como um corpo de conhecimento plural e extenso em contribuições, isso porque esse corpo teórico tem sido defendido como essencialmente empírico e pragmático.

Apesar de seu potencial, não há um consenso quanto à organização conceitual interna da teoria da gestão comparativa, uma vez que esta possui uma diversidade de abordagens, as quais são pouco exploradas por estudiosos que se aplicam a melhor compreender os temas vinculados a esse corpo teórico. Essa constatação justifica as dificuldades que os estudos teórico-empíricos orientados pela teoria da gestão comparativa têm para abordar e analisar temas mais polêmicos e complexos. 0 reconhecimento dessas fragilidades motivou a redação deste ensaio teórico, que tem como objetivo propor, por meio de contribuições teóricas relevantes, quatro abordagens de estudo representativas da teoria da gestão comparativa, sendo elas: escola colonialista da teoria da gestão comparativa, escola neocolonialista da teoria da gestão comparativa, escola pós-colonialista amena da teoria da gestão comparativa e escola pós-colonialista crítica da teoria da gestão comparativa.

Para um melhor aprofundamento do tema em discussão, o presente ensaio foi dividido nas seguintes seções: inicialmente, em um período que começa no colonialismo e que culmina no neocolonialismo, a teoria da gestão comparativa é contextualizada historicamente e demarcada em relação aos seus principais temas de estu- do; posteriormente, as bases colonialistas da teoria da gestão comparativa são questionadas com base em reivindicações pós-colonialistas; em sequência, são apresentadas e classificadas quatro escolas para a teoria da gestão comparativa; e, finalmente, o artigo é encerrado com algumas considerações e reflexões. Para a proposição das quatro escolas, como abordagens de estudo representativas da teoria da gestão comparativa, foram selecionadas as seguintes vertentes de estudo do corpo teórico em perspectiva: as escolas colonialistas e pós-colonialistas (Boyacigiller \& Adler, 1991; Harding, 1998; Neghandi, 1975; Schollhammer, 1969; Westwood, 2001, 2004), as segmentações teórico-abstrata e empírica (Schollhammer, 1973) e as abordagens socioeconômica, ecológica e comportamental (Neghandi, 1975; Schollhammer, 1969). A seleção dessas contribuições foi necessária para garantir a viabilidade das discussões propostas por meio deste ensaio teórico.

\section{CONTEXTUALIZAÇÃO HISTÓRICA E DEMARCAÇÃO TEMÁTICA: DO COLONIALISMO AO NEOCOLONIALISMO}

Os fatos históricos que originaram os debates sobre a teoria da gestão comparativa, cujo conjunto é denominado, neste ensaio teórico, escola colonialista da teoria da gestão comparativa, ocorreram nos séculos XV e XVI, quando o capitalismo mercantil inspirou alguns Estados Absolutistas europeus a empreender estratégias de colonização sobre territórios espalhados ao redor do mundo. As bases primitivas desse corpo teórico estão alicerçadas em reflexões e investigações sobre a relação de subordinação imposta pelas nações europeias, também denominadas metrópoles, aos territórios colonizados, também denominados colônias (Gilmartin, 1994; Harding, 1996). As decisões relacionadas aos projetos de colo- nização eram tomadas nas metrópoles europeias por uma tríade elitista, composta pelos seguintes elementos: o rei, como representante do Estado Absolutista; a nobre$z a$, como defensora dos tradicionalismos inerentes ao Estado Absolutista; e a burguesia, ou os novos-ricos, como financiadora desse Estado Absolutista (Cooke, 2003a; Reingold \& Rothenberg, 1987).

Cabia à tríade elitista a responsabilidade de transferir os ideais metropolitanos aos territórios colonizados, por meio da instalação e da disseminação de valores culturais, de comportamentos sociais e de instituições administrativas de referência (tais como a Igreja e o Estado). Essa sobreposição cultural buscava legitimar a autoridade jurídica das metrópoles europeias sobre os territórios colonizados, validar a liberdade comercial europeia de explorar livremente os recursos naturais e a mão de obra das pessoas dessas colônias e esclarecer uma autorização divina que justificasse esse relacionamento tão materialista e ofensivo para com as pessoas e os territórios colonizados. 0 projeto de colonização nunca ficou retido à limitação geográfica de posse de terras, mas, desde o seu início, procurou um elemento pelo qual pudesse estender a força colonizadora da metrópole para diferentes instâncias (Basalla, 1967; Prasad, 2003; Said, 1993).

0 real propósito colonial-mercantilista era ofuscado por um falso ideal metropolitano que compartilhava fins morais e religiosos, tais como os de educar, de instruir, de capacitar e de salvar espiritualmente os habitantes das colônias, denominados na literatura como "os outros". Esses "outros", apesar de constituírem-se como categoria de análise relevante para a teoria da gestão comparativa, representam uma invenção da ciência moderna ocidental. Cada metrópole, por uma atuação intensa de sua respectiva tríade elitista, ao julgar a população colonizada (ou seja, "os outros") como desprovida de conhecimento, de contato com o divino e de orientação econômica, des- 
prezava o patrimônio sociocultural dessas comunidades (tribos indígenas, organizações coletivas alternativas etc.) e determinava a multiplicação de comportamentos que deveriam fazer "dos outros" (colonizados) imagem e semelhança dos metropolitanos. "Os outros", portanto, apenas existem a partir de uma comparação com um ideal metropolitano (Cooke, 2003b; Fabian, 2001; Goodman \& Moore, 1972).

Essa invenção discursiva decorrente da ação colonizadora foi manifestada em um exercício comparativo orientado pelo interesse de reificar o colonizado a partir de um padrão metropolitano que contribuiu com o desenvolvimento da ciência do norte (também denominada epistemologia do norte) e a afiliação dessa ciência com a antropologia (Abdel-Malek, 1971; Houtondji, 1997; Santos, 2007). A partir dessa relação, pretendia-se encontrar justificativas racionais e maneiras sistemáticas de se dominarem "os outros". Por diversas estratégias de investigação e de exploração, "os outros" começaram a se transformar em um fenômeno mais compreensível para o conjunto de conhecimentos metropolitanos e, por consequência, aos sistemas de administração das colônias (Asad, 1973; Redding, 1994).

A escola colonialista da teoria da gestão comparativa compreende a gestão como a administração dos territórios colonizados, ou seja, a sistematização e o acompanhamento de atividades como a exploração dos recursos naturais e a utilização da mão de obra das pessoas (“dos outros") dessas colônias, a partir de ideais metropolitanos. Metrópole e colônia, portanto, representam as organizações estudadas pela escola coIonialista da teoria da gestão comparativa. Por consequência dessa racionalidade administrativa, a comparação aplicada pelas bases primitivas da teoria da gestão comparativa (entre metrópole e colônia) compartilhava uma inspiração econômica e um propósito de dominação. A partir das características históricas descritas, considera-se que a escola colonialista da teoria da ges- tão comparativa emerge de duas estruturas mutuamente constitutivas, o capitalismo, como sistema econômico, e o colonialismo, como uma das práticas econômicas defendidas pela versão mercantilista do capitalismo (Cooke, 2003c; Jack \& Westwood, 2009; Redding, 2005).

Essa relação econômica, marcada por uma imposição metropolitana e por uma submissão colonial, propiciou o desenvolvimento de conhecimentos científicos, de saberes administrativos, de práticas comerciais e de argumentos antropológicos, que, em conjunto, por inspiração do capitalismo e pela aplicação do colonialismo, contribuíram para alicerçar os ideais da modernidade. Com o decorrer da história, o colonialismo perdeu o seu destaque como prática econômica capitalista, e, por consequência, a oficialidade da relação metrópole-colônia foi gradativamente desconstruída. No entanto, observa-se, no cenário macroeconômico contemporâneo, uma nova organização de relações marcadas por imposições e submissões, que é aquela estabelecida entre o centro euro-americano e os demais países do mundo, rotulada, neste ensaio teórico, como escola neocolonialista da teoria da gestão comparativa (Engwall, 1996; Springhall, 2001; Westwood \& Jack, 2007).

A escola neocolonialista da teoria da gestão comparativa está fundamentada por uma ideologia neoliberal, compreendida como um aprimoramento do sistema capitalista sobre o Estado Democrático Liberal e sobre o Estado Democrático Social, e que tem como propósito reduzir a função social e priorizar a função gerencial do Estado ao transformar o nacionalismo, o socialismo e o ambientalismo como objetivos subordinados ao liberalismo. Por essa afiliação com o Estado Neoliberal, o neocolonialismo é compreendido pelos mais críticos da escola colonialista da teoria da gestão comparativa como um colonialismo contemporâneo, no qual o centro euro-americano representa a metrópole e os demais países subordinados a esse centro figuram a fun- ção de colônias modernizadas (Alvares, 1988; Escobar, 1995; Guha \& Spivak, 1988).

Apesar das críticas, é necessário evocar o que diferencia o neocolonialismo e o colonialismo como escolas da teoria da gestão comparativa. Nesse movimento mais contemporâneo do corpo teórico em perspectiva, a dominação territorial de uma nação sobre a outra, ou seja, a colonização territorial, não existe mais. Por consequência, o centro euro-americano, em relação aos países subordinados a ele, não possui autoridade jurídica (direta), não participa de uma liberdade comercial (não consentida) de explorar livremente os recursos naturais e a mão de obra e não tem um compromisso (declarado) de cunho religioso (Betts, 2004; Chaterjee, 1986). A diferença, portanto, está na ausência de dominação territorial. Mas o que faz, de acordo com os mais críticos, do neocolonialismo um colonialismo contemporâneo e dos países subordinados ao centro euro-americano colônias modernizadas?

A resposta está no que Thrift (2005) denominou "circuito cultural do capitalismo", entendido como o poder que o sistema capitalista tem de tornar suas teorias e descrições do mundo efetivas, por meio de estratégias de produção prescritivas e proposicionais que ofereçam às sociedades novas máquinas e novos corpos, os quais são simultaneamente disseminados e aceitos por um discurso assumido como legítimo. Para o neocolonialismo, o interesse econômico está em acessar o máximo de sociedades possíveis por meio de um aliciamento cultural que dispensa uma prévia dominação territorial (Weinshall, 1977; Westwood, 2004). O Estado Neoliberal subsidia o circuito cultural do capitalismo na medida em que permite, de acordo com as proposições de Deleuze (1992), o rompimento com as sociedades disciplinares e a ascensão das sociedades de controle.

As sociedades disciplinares privilegiam o confinamento institucional (família, escola, fábrica, hospital, prisão, igreja) e vi- 
gilância contínua. Já as sociedades de controle reconhecem que o mundo é inconsistente, complexo, paradoxal, confuso e, por consequência, exigente de um novo gerencialismo, marcado por um controle de curto prazo, de rotação rápida, contínuo e ilimitado (Deleuze, 1992; Fontenelle, 2012). 0 que importa para o neocolonialismo não é a dominação como causa, mas o controle por meio de dispositivos culturais que, por alterarem-se constantemente com o passar do tempo, tentam viabilizar o contínuo acesso às mais diversas sociedades (Cameron \& Palan, 2004; Kelly, 1999). O símbolo não é mais o Estado-Nação, mas as organizações que declaradamente possuem fins econômicos e que contribuem para a perpetuação do circuito cultural capitalista em diferentes países (Joynt, 1985; Tucker, 1999).

Qualquer organização que tem o lucro como um objetivo a ser alcançado pelo foco do seu negócio contribui para a manutenção do circuito cultural capitalista e para a legitimação da sociedade de controle. Da população dessas organizações, interessam à escola neocolonialista da teoria da gestão comparativa aquelas que atuam em mais de um país, contanto que as matrizes estejam localizadas no centro euro-americano e as filiais estejam espalhadas em países externos a esse eixo dominante. 0 exercício comparativo aplicado no neocolonialismo pretende compreender, na relação matriz e filial, as interferências e as contribuições provocadas pelas similaridades e pelas diferenças socioculturais nos processos de gestão e nas operações das empresas multinacionais (Scholte, 2000; Tsui, 2007).

Para a escola neocolonialista da teoria da gestão comparativa, a metrópole personifica-se nas empresas multinacionais, cujas matrizes estão em território euro-americano. A disciplina imposta pela colonização territorial e pela dominação sociocultural do colonialismo é substituída, no neocolonialismo, por um controle fundamentado pela contribuição (in)di- reta dessas empresas multinacionais com o circuito cultural capitalista. Os neocolonialistas, no que se refere ao estudo das diversas relações entre matriz e filial, possuem alguns propósitos, entre os quais se destacam os seguintes: entender as interações e as negociações das empresas multinacionais euro-americanas com as instituições governamentais dos países onde suas filiais serão e/ou estão instaladas; investigar o posicionamento dessas multinacionais sobre as metas de nacionalismo econômico dos países que amparam o funcionamento de suas respectivas filiais; e analisar os impactos, as causas e as consequências das diferenças culturais vivenciadas nos países externos ao centro euro-americano e manifestadas no exercício da gestão das filiais (Jack \& Westwood, 2009; Neghandi, 1975; Schollhammer, 1975; Westwood, 2001, 2004).

Esses propósitos neocolonialistas dedicam-se ao interesse de identificar, por meio de uma melhor compreensão do contexto socioeconômico e do cenário sociocultural das regiões nas quais as filiais estão instaladas, os mecanismos legais, comportamentais e socioeconômicos que permitam a inserção dos ideais culturais euro-americanos nessas comunidades. Por suas diversas atuações estratégicas, essas organizações internacionalizadas infiltram-se em comunidades distribuídas ao redor do mundo e assumem representatividade política e econômica, que lhes concede: poder para compartilhar e influenciar o poder jurídico dessas localidades; liberdade para empreender negócios de diversas naturezas e empregar pessoas; e oportunidade de disseminar um ideal sociocultural mais afim com os padrões euro-americanos. A dominação não é uma causa maior para o neocolonialismo, mas uma consequência proveniente de um controle sociocultural movido pelo interesse econômico de se internacionalizar uma cultura dominante. Ainda que por práticas diferenciadas, o neocolonialismo também busca a coloniza- ção “dos outros” (Levy, 2008; Meyer \& Geschiere, 1999; Thomas, 1994).

“Os outros”, em relação à escola neocolonialista da teoria da gestão comparativa, são todos aqueles que sofrem, direta ou indiretamente, impacto da ação administrativa de empresas multinacionais que participam do movimento econômico de disseminar e legitimar, em âmbito internacional, o circuito cultural capitalista. Para o neocolonialismo, “os outros” estão em qualquer coletividade que ainda não tenha entrado em contato e/ou que ainda não tenha cedido às pressões internacionais de se adequar aos padrões culturais euro-americanos. "Os outros", para as matrizes de empresas multinacionais euro-americanas, são: as filiais, os funcionários das filiais, os consumidores dos países das filiais, os fornecedores regionais que atendem as filiais, o governo dos países das filiais, a sociedade dos países das filiais, os movimentos sociais dos países das filiais (ONGs, sindicatos, associações) e as instituições representativas dos países das filiais (escolas, universidades, bibliotecas, hospitais, igrejas, organizações midiáticas etc.) (Appadurai, 1990; Hoogvelt, 2001; Westwood, 2001).

A escola neocolonialista da teoria da gestão comparativa compreende a gestão como a administração de empresas multinacionais que tenham suas matrizes localizadas no centro euro-americano e que contribuam para a inserção e perpetuação do circuito cultural capitalista nos territórios externos a esse eixo dominante. Matriz e filial são as organizações estudadas na escola neocolonialista da teoria da gestão comparativa. 0 perfil estratégico e administrativo das relações estabelecidas entre matriz e filial fundamenta uma comparação neocolonialista que compartilha uma inspiração econômica e um ideal de controle que, em conjunto, pretendem uma dominação sociocultural. A partir das características apresentadas, argumenta-se que a teoria da gestão comparativa neocolonialista 
está alicerçada em duas estruturas mutuamente constitutivas, o capitalismo, como sistema econômico, e a internacionalização de empresas, como uma das práticas econômicas defendidas pela versão neoliberalista do capitalismo Jack \& Westwood, 2009; Neghandi, 1975; Schollhammer, 1975; Westwood \& Jack, 2007).

Após esta breve contextualização histórica sobre a teoria da gestão comparativa, que se iniciou no colonialismo e culminou no neocolonialismo, por meio da qual se demarcaram os principais temas abordados e amparados por esse corpo teórico, passa-se para uma reflexão crítica desses fundamentos a partir de uma análise pós-colonialista.

\section{DESCOLONIZANDO A TEORIA DA GESTÃO COMPARATIVA: O PÓS-COLONIALISMO COMO UMA TENTATIVA DE SUPERAÇÃO}

As características epistemológicas e metodológicas predominantes da teoria da gestão comparativa têm suas raízes nas práticas de colonização realizadas, principalmente, pelas nações do continente europeu sobre todo o mundo. Essa relação, em que uma nação principal (metrópole) dominava a outra (colônia), caracterizou o início das análises comparativas, as quais estão amparadas pela escola colonialista da teoria da gestão comparativa (Prasad, 2003; Westwood, 2001).

Com o decorrer do tempo e, principalmente, com a perpetuação do capitalismo, essa colonização foi substituída por uma submissão econômica de inúmeros países em relação ao centro euro-americano. As pesquisas sobre a teoria da gestão comparativa são organizadas, contemporaneamente, em um projeto euro-americano, o qual possui os propósitos de apreender, prever e controlar os sistemas de desenvol- vimento econômico, tecnológico e produtivo de outras nações, ou seja, uma nova roupagem da escola colonialista, denominada escola neocolonialista da teoria da gestão comparativa (Bass, 1965; Beaty \& Mendenhall, 1990).

Os estudos vinculados à escola neocolonialista da teoria da gestão comparativa, em sua grande maioria, são conduzidos por e para esse centro euro-americano. Essas investigações buscam, a partir desse centro, analisar outras nações (neste caso, não mais colônias, mas países subordinados a esse centro - também denominados “outros") em suas práticas de gestão de negócios para que, após essa análise, essas mesmas práticas sejam reificadas de acordo com os padrões centrais. A padronização, por sua vez, facilita, para o centro euro-americano, o desenvolvimento de parcerias comerciais, a criação de alianças estratégicas e a disseminação desse modelo gerencialista essencialmente empirista (Punnett \& Shenkar, 1996; Westwood, 2004).

A reificação "dos outros" (nações externas ao centro Euro-Americano) faz com que eles sejam classificados como disfuncionais, irracionais, ineficientes, incompetentes, retrógrados, e assim por diante. Esse cenário perpetua estratégias de relações internacionais impositivas, pelas quais esse centro euro-americano assume a liderança nas orientações e transformações ocorrentes nos países em desenvolvimento, tais como alteração nas estruturas sociais, nos valores e nos padrões comportamentais de diversas sociedades (Harbison \& Myers, 1959; Prasad, 1997).

A escola neocolonialista da teoria da gestão comparativa, a partir da continuidade dos propósitos colonialistas, contribui com o equívoco acadêmico de se considerarem as premissas positivistas as mais coerentes para analisar comparativamente as diferentes relações firmadas entre o centro euro-americano e "os outros". O equívoco justifica-se pelo fato de não ser pos- sível aceitar as contribuições advindas das práticas dominantes como representativas de um conhecimento universal, unificado e inquestionável. 0 ideal emancipatório da escola pós-colonialista da teoria da gestão comparativa surge como um questionamento à universalidade desse conhecimento, ao classificá-lo apenas como um conhecimento que foi desenvolvido em um determinado momento histórico, sob determinadas regras sociais e com características sistêmicas particulares (Dupre, 1993; Harding, 1998).

0 pós-colonialismo, portanto, com 0 desenvolvimento da teoria da gestão comparativa, passou a representar, mais do que o rompimento histórico de relações outorgadas entre metrópole (centro Euro-Americano) e colônia (“os outros”), uma abordagem de comparação em que as partes analisadas são equiparadas em relevância e potencialidade de contribuição. O questionamento pós-colonialista representa simultaneamente uma evolução e uma alteração do fenômeno de estudo da teoria da gestão comparativa. Enquanto o colonialismo prioriza a padronização a partir da metrópole e, na contemporaneidade, o neocolonialismo assume o centro euro-americano como um ideal de referência, o pós-colonialismo destitui a concepção de padrão, de ideal e de referência e passa seu foco analítico para nações, regiões, coletividades, organizações e indivíduos que estão fora de um centro específico, ou seja, para "os outros".

A escola colonialista exalta a metrópole e a escola neocolonialista privilegia o centro euro-americano, por acreditarem que "os outros" simbolizam ameaça, perigo e patologia social. 0 padrão dominante, portanto, foi estabelecido para amenizar as ameaças, extinguir os perigos e educar "os outros". A lapidação na significação "dos outros", para a teoria da gestão comparativa pós-colonialista, o constitui como um fenômeno: múltiplo; simultaneamente comparável e específico; com comportamentos 
imprevisíveis em relação aos diferentes temas abordados pela teoria da gestão comparativa; com comportamentos que influenciam pessoas, organizações e sociedades; com uma natureza essencialmente interdisciplinar; e, expressado pelo significado estrutural e coletivo dos atores pesquisados (Boyacigiller \& Adler, 1991; Parry, 1987; Prasad, 1997; Redding, 1994).

Por meio das concepções colonialista e neocolonialista sobre "os outros", observa-se uma natureza objetivista, desenvolvida por meio de abordagens dedutivas, testes de hipóteses e de análises estatísticas, os quais não compactuam com o pluralismo ideológico e valorizam discussões orientadas por um funcionalismo estrutural (Thomas, 1994). A escola pós-colonialista da teoria da gestão comparativa, por sua vez, rompe com hegemonias teóricas, metodológicas, institucionais e políticas que circundam os contextos investigativos desse corpo teórico. As reivindicações pós-colonialistas exigem consideração aprofundada de preceitos ontológicos, epistemológicos, metodológicos, políticos, éticos e institucionais, os quais, em conjunto, defendem a necessidade de as burocracias, as culturas, as comunicações linguísticas e as manifestações comportamentais emanciparem-se da influência euro-americana Jack \& Westwood, 2009; Smith, 1999).

A escola pós-colonialista da teoria da gestão comparativa acredita em uma realidade socialmente construída, dependente dos indivíduos e das coletividades dos indivíduos e avessa a uma padronização que sufoque, ou que relativize, as vozes de grupos menos favorecidos. O pós-colonialismo adere ao desafio de superação do positivismo por um caminho subjetivista de compreensão da realidade e de construção do conhecimento marcado pelas seguintes características: investigar e criticar os pressupostos universais que orientam as teorias e metodologias dominantes nas pesquisas sobre internacionalização; permitir que ideologias observadas fora da perspectiva euro-americana sejam consideradas em um mesmo patamar de relevância; desafiar a dependência criada em relação aos procedimentos quantitativos de pesquisa; trabalhar em prol de um conhecimento científico que seja epistemologicamente plural e interdisciplinar; valorizar pesquisas teóricas elaboradas a partir das perspectivas críticas que permitam identificar as tensões políticas e filosóficas existentes entre positivismo e outras epistemologias; e fomentar estudos que sejam conduzidos por abordagens mais dialógicas, que concedam à teoria da gestão comparativa a capacidade de reconhecer a diversidade das identidades sociais e das relações de poder existentes em cada um dos territórios, organizações e ambientes pesquisados por esse corpo teórico (Peterson, 2001; Pfeffer, 2005; Tsui, 2007).

A teoria da gestão comparativa pós-colonialista, em síntese, pretende uma descolonização da teoria da gestão comparativa colonialista e neocolonialista, mais especificamente em seus aspectos epistemológicos, metodológicos e conceituais. Propõe-se que o ideal emancipatório e o perfil questionador dos pós-colonialistas se repartam em dois caminhos: (1) escola pós-colonialista crítica; e (2) escola pós-colonialista amena. 0 primeiro questiona o sistema atual e denuncia suas falhas como as principais causas de todas as crises e diferenças socioeconômicas contemporâneas. 0 segundo também questiona o sistema atual, mas por um caminho mais ameno, justificado pela apresentação de soluções para cada uma das patologias sociais, culturais e econômicas criticadas. Essa segmentação tem origem epistemológica e é diferenciada em relação à proposição ou a não proposição de soluções para as críticas levantadas (Balbinot \& Borim-De-Souza, 2013; Harding, 1998; Neghandi, 1975; Schollhammer, 1969; Westwood, 2001).

Nesta seção, as bases colonialistas da teoria da gestão comparativa foram ques- tionadas a partir de reivindicações pós-colonialistas. A contextualização histórica que apresentou o colonialismo e o neocolonialismo e a análise crítica que introduziu o pós-colonialismo subsidiam o debate para a proposição de quatro escolas de estudo para a teoria da gestão comparativa.

\section{APRESENTAÇÃO E CLASSIFICAÇÃO DAS ESCOLAS DA TEORIA DA GESTÃO COMPARATIVA}

A proposição das quatro escolas como abordagens de estudo representativas da teoria da gestão comparativa ocorreu a partir das seguintes vertentes de estudo do corpo teórico em perspectiva: as escolas colonialista e pós-colonialistas (Boyacigiller \& Adler, 1991; Harding, 1998; Neghandi, 1975; Schollhammer, 1969; Westwood, 2001, 2004), as segmentações teórico-abstrata e empírica (Schollhammer, 1973) e as abordagens socioeconômica, ecológica e comportamental (Neghandi, 1975; Schollhammer, 1969). A seleção dessas contribuições foi necessária para garantir a viabilidade das discussões propostas por meio deste ensaio teórico.

Em sua vertente mais crítica, a escola pós-colonialista constrói-se por tentativas de fundamentar a teoria da gestão comparativa por meio da proposição de modelos teóricos, estruturas conceituais e tipologias específicas para um posterior desenvolvimento e teste de hipóteses. Tais interesses permitem classificar a escola pós-colonialista crítica como teórico-abstrata. As escolas colonialista e neocolonialista, pelo caráter mais objetivista, promovem pesquisas que buscam explicar, avaliar e acumular os dados coletados pelas pesquisas empíricas, o que permite classificá-las como escolas empíricas. Já a escola pós-colonialista amena aborda investigações que são simultaneamente críticas e propositivas, ou seja, 
desenvolve pesquisas que, ao mesmo tempo, têm origem a partir de uma falha ou de uma patologia sistêmica, as quais são acompanhadas de uma proposição de aplicação empírica, bem por isso, essa escola é tanto teórico-abstrata quanto empírica. Essa classificação flexível da escola pós-colonialista amena justifica-se por ela estar em um espaço de discussão que resgata princípios radicalmente subjetivistas e, também, por depender de procedimentos empíricos essencialmente objetivistas (Beaty \& Mendenhall, 1990; Bhatt \& Miller, 1983; Hoogvelt, 2001; Schollhammer, 1975).

Por possuir raízes epistemológicas positivistas, as escolas colonialista e neocolonialista têm, entre os seus principais compromissos, a necessidade de orientar seus estudos para o desenvolvimento de contribuições que culminem, ou, ainda, que contribuam, para o desenvolvimento econômico do centro euro-americano. Suas análises partem do pressuposto de que a prática da gestão constitui a característica mais crítica para que melhores índices de desenvolvimento econômico sejam conquistados. 0 gestor assume um comportamento determinístico, ou seja, age somente em resposta às demandas apresentadas pelo ambiente. A gestão, apesar de ser considerada um dos principais veículos de mudança, é reconhecida como apenas uma das partes que integram todo o sistema socioeconômico. Esse comprometimento com o desenvolvimento econômico permite relacionar as escolas colonialista e neocolonialista com a abordagem socioeconômica (Neghandi, 1975; Schollhammer, 1969).

Em contrapartida ao enfoque econômico das escolas colonialista e neocolonialista, está a priorização comportamental da escola pós-colonialista crítica. Enquanto as escolas colonialista e neocolonialista validam, por meio da abordagem socioeconômica, que o gestor age de acordo com demandas ambientais, muitas delas inesperadas, a escola pós-colonialista crítica prioriza os comportamentos específi- cos desses mesmos gestores, os quais são assumidos como relevantes por exercerem suas funções de gestão em diferentes contextos culturais. As motivações desses gestores para a realização de ações gerenciais, bem como os relacionamentos profissionais que circundam o exercício da gestão, são consideradas, uma vez que tais características contribuem para a compreensão de como ocorre a interação entre esse indivíduo (representante da organização e da coletividade de outros indivíduos) e a sociedade. A fundamentação reacionária e subjetivista da escola pós-colonialista crítica defende a contribuição dos indivíduos para a construção da realidade, bem por isso, a importância da abordagem comportamental para a elaboração de seus pressupostos teórico-abstratos (Harding, 1998; Neghandi, 1975; Schollhammer, 1969; Westwood, 2004).

A escola pós-colonialista amena, por sua vez, alia a necessidade de um desenvolvimento econômico, ao ressaltar a relevância de analisar o ambiente externo para a eficiência da firma, com a importância de se compreender o comportamento do gestor, ao validar os recursos e as competências como diferenciais indispensáveis para o sucesso da firma. Esse contrabalanceamento de interesses é tratado pela abordagem ecológica da teoria da gestão comparativa, que busca isolar variáveis do ambiente externo por meio das quais similaridades e diferenças de contextos gerenciais de diversos países são analisadas. 0 negócio em si é observado como um sistema ecológico, no qual os fatores externos possuem impacto determinante sobre o desempenho da firma e sobre a eficiência econômica do sistema. O compartilhamento do enfoque entre gestor (indivíduo), organização (coletividade de indivíduos) e ambiente (coletividade de organizações) permite a identificação de vários componentes ecológicos, tais como as características sociológicas, políticas e econômicas de regiões, características essas assumidas como impeditivas ou poten- cializadoras de uma efetiva prática gerencial (Hannan \& Freeman, 1977; Katz, 2001; Neghandi, 1975; Schollhammer, 1969).

As discussões teóricas apresentadas indicam que os estudos sobre a teoria da gestão comparativa diferenciam-se quanto à concepção e ao tratamento a ser concedido "aos outros" (reificação ou valorização), ao propósito de investigação (teórico-abstrato e/ou empírico) e ao enfoque analítico (desenvolvimento econômico, contribuição comportamental dos indivíduos e relação entre gestor, organização e ambiente). A partir das relações entre essas segmentações da teoria da gestão comparativa, propõem-se quatro abordagens representativas para esse corpo teórico: escola colonialista da teoria da gestão comparativa, escola neocolonialista da teoria da gestão comparativa, escola pós-colonialista crítica da teoria da gestão comparativa e escola pós-colonialista amena da teoria da gestão comparativa.

As escolas colonialista e neocolonialista da teoria da gestão comparativa defendem a reificação "dos outros” e desenvolvem pesquisas que buscam explicar, avaliar e acumular dados coletados por pesquisas empíricas inseridas em estudos que contribuem com o desenvolvimento econômico da metrópole (colonialismo) e do centro euro-americano (neocolonialismo). A escola pós-colonialista crítica defende a valorização e a emancipação “dos outros” em relação ao padrão euro-americano, e, por tal razão, se constrói por tentativas de fundamentar a teoria da gestão comparativa por meio da proposição de modelos teóricos, estruturas conceituais e tipologias específicas que privilegiam analisar a contribuição dos indivíduos (mais especificamente, dos gestores) para a construção social da realidade. A escola pós-colonialista amena, ao compartilhar o enfoque de seus estudos entre o gestor (indivíduo), a organização (coletividade de indivíduos) e o ambiente (coletividade de organizações), incentiva a valorização “dos outros” e ampara a reali- 
zação de pesquisas que são, simultaneamente, um espaço para reflexões teóricas aprofundadas e dependentes de procedimentos empíricos de investigação.

\section{CONSIDERAÇÕES E REFLEXÕES}

Este ensaio teórico, apesar de encerrar suas discussões na apresentação de quatro escolas da teoria da gestão comparativa, não limita suas contribuições ao escopo de páginas que amparam o corpo deste texto. 0 objetivo complementar deste estudo é fomentar discussões acadêmicas sobre a teoria da gestão comparativa. Tais discussões contribuirão para que a teoria da gestão comparativa continue a ser (re)apresentada como um corpo teórico plural e extenso em contribuições.

A apresentação das escolas da teoria da gestão comparativa demonstra uma evolução nos estudos e nas conceituações desse corpo teórico. Se comparados os propósitos de análise das escolas colonialista e neocolonialista com os interesses das escolas pós-colonialistas, percebe-se que “o outro", gradativamente, deixa de ser essencializado e passa a ser compreendido em coerência com o contexto no qual exerce suas diversas atividades sociais. Essa valorização “do outro" repete-se nas demais abordagens discutidas, pois a prioridade de análise sai de uma esfera puramente econômica, passa por uma ótica integracionista e recai na relevância das motivações e relacionamentos profissionais dos gestores (considerados agentes sociais) inseridos em diferentes contextos organizacionais.

As escolas pós-colonialistas, em síntese, superam os conhecimentos que principiaram a teoria da gestão comparativa por encontrar "no outro", ou "nos outros", a fonte de contribuições que fazem desse corpo teórico um diferencial. Contempla-se um rompimento com a teoria da gestão comparativa colonialista e neocolonialista por meio de um questionamento das tradições funcionalistas e empíricas, o qual cobra um reposicionamento ontológico e epistemológico que fundamente, com coerência, as reivindicações e as proposições da teoria da gestão comparativa pós-colonialista, em suas escolas amena e crítica.

\section{REFERÊNCIAS}

Abdel-Malek, A. (1971). L'avenir de la théorie sociale. Cahiers Internationaux de Sociologie, 50, $23-40$.

Adler, N. J. (1983). Cross-cultural management research: the ostrich and the trend. Academy of Management Review, 8(2), 226-232.

Adler, N. J., Doktor, R., \& Redding. (1986). From the Atlantic to the Pacific century: cross-cultural management reviewed. Journal of Management, 12(2), 295-318.

Alton, A. J. (1969). Comparative management: how useful to the practioner? Management International Review, 9(1), 3-11.

Alvares, C. (1988). Science, colonialism and violence: a Luddite view. In A. Nandy (Ed.) Science, hegemony and violence (pp. 68-112). Bombay: Oxford University Press.

Appadurai, A. (1990). Disjuncture and difference in the global culture economy. Theory, culture and society, 7(2), 295-310.

Asad, T. (Ed.)(1973). Anthropology and the colonial encounter. London: Ithaca Press.

Balbinot, Z., \& Borim-De-Souza, R. (2013). A cultura como tema de interesse para a Teoria da Gestão Comparativa. Interações, 14(2), 279-294.

Basalla, G. (1967). The spread of western science. Science, 156(3775), 611-622.

Bass, B. M. (1965). Organizational psychology. Boston: Allyn and Bacon.

Beaty, D. T., \& Medenhall, M. (1990). Theory building in international management: an archival review and recommendations for future research. Proceedings of Academy of Management Annual Meeting, San Francisco, 50.

Betts, R. F. (2004). Decolonization. London and New York: Routledge.

Bhatt, B. J., \& Miller E. L. (1983). A framework for upgrading comparative management research. Asia Pacific Journal of Management, 1(1), 26-35

Boyacigiller, N., \& Adler, N. J. (1991). The parochial dinosaur: organizational science in a global context. Academy of Management Review, 16(2), 262-290.
Cameron, A., \& Palan, R. (2004). The imagined economies of globalization. London: Sage.

Chaterjee, P. (1986). Nationalist thought and the colonial world: a derivative discourse. London: Zed Books.

Cooke, B. (2003a). A new continuity with colonial administration: participation in development management. Third World Quarterly, 24(1), 47-61.

Cooke, B. (2003b). Managing organization culture and imperialism. In A. Prasad (Ed.). Postcolonial theory and organizational analysis: a critical engagement (pp. 75-94). New York: Palgrave Macmillan.

Cooke, B. (2003C). The denial of slavery in management studies. Journal of Management Studies, 40(8), 1895-1918.

Deleuze, G. (1992). Conversações. São Paulo: Editora 34.

Dupre, J. (1993). The disorder of things: metaphysical foundations for the disunity of science. Cambridge: Harvard University Press.

Engwall, L. (1996). The Vikings versus the world: an examination of Nordic business research. Scandinavian Journal of Management, $12(4), 425-436$.

Escobar, A. (1995). Encountering development: the making and unmaking of the third world. Princeton: Princeton University Press.

Fabian, J. (2001). Time and the other: how an thropology makes its object. New York: Colum bia University Press.

Fontenelle, I. A. (2012). Para uma crítica ao discurso da inovação: saber e controle no capitalismo do conhecimento. RAE-Revista de Administração de Empresas, 52(1), 100-108.

Gilmartin, D. (1994). Scientific empire and imperial science: colonialism and irrigation in the Indus Basin. The Journal of Asian Studies, 53(4), 1127-1149.

Goodman, P. S., \& Moore, B. E. (1972). Critical issues of cross cultural management research. Human Organization, 31(1), 39-45.

Guha, R., \& Spivak, G. C. (1988). Selected subaltern studies. New York: Oxford University Press.

Hannan, M. T., \& Freeman, J. (1977). The population ecology of organizations. American Journal of Sociology, 82(5)929-964.

Harbison, F., \& Myers, C. A. (1959). Management and the industrial world: an international analysis. New York: McGraw-Hill.

Harding, S. (1996). European expansion and the organization of modern science: isolated or linked historical processes. Organization, 3(4), 497-509. 
Harding, S. (1998). Is science multicultural? Postcolonialisms, feminisms and epistemologies. Indianapolis: Indiana University Press.

Hoogvelt, A. (2001). Globalization and the postcolonial world. London: Palgrave.

Hountondji, P. J. (1997). Endogenous knowledge: research trails. Dakar: Codesria.

Jack, G, \& Westwood, R. (2009). International and cross-cultural management studies: $a$ postcolonial reading. London: Palgrave MacMillan.

Joynt, P. (1985). Cross-cultural management: the cultural context of micro and macro organizational variables. In P. Joynt \& M. Warner (Eds.). Managing in different cultures (pp. 5768). Oslo: Universitetsforlaget.

Katz, C. (2001). On the grounds of globalization: a typography for feminist political engagement. Signs, 26(1), 1213-1234.

Kelley, L, \& Worthley, R. (1981). The role of culture in comparative management: a cross-culture perspective. Academy of Management Journal, 24(1), 164-173.

Kelly, J. D. (1999). Time and the global: against the homogeneous, empty communities in contemporary social theory. In B. Meyer \& P. Geschiere (Eds.). Globalization and identity: dialectics of flow and closure (pp. 239-272). Oxford: Blackwell.

Kochan, T. A. (1983). Review of industrial democracy in Europe. Administrative Science Quarterly, 28(4), 629-634.

Levy, D. (2008). Political contestation in global production networks. Academy of Management Review, 33(4), 943-963.

Meyer, B, \& Geschiere, P. (Eds.). (1999). Globalization and identity: dialectics of flow and closure. Oxford: Blackwell.

Neghandi, A. R. (1975). Comparative management and organization theory: a marriage needed. Academy of Managament Journal, 18(2), 334-344.

Parry, B. (1987). Problems in current theories of colonial discourse. Oxford Literary Review, 13(1), 25-58.

Peterson, M. F. (2001). International collaboration in organizational behavior research. Journal of Organizational Behavior, 22(1), 59-81.
Pfeffer, J. (2005). Why do bad management theories persist? A comment on Ghoshal. Academy of Management Learning and Education, 4(1), 96-100.

Prasad, A. (1997). The colonizing consciousness and representations. In P. Prasad, A. J. Mills, M. Elmes \& A. Prasad A. (Eds.). Managing the organizational melting pot: dilemmas of workplace diversity (pp. 285-311). London: Sage.

Prasad, P. (2003). Postcolonial theory and organizational analysis: a critical engagement. New York: Palgrave.

Punnett, B. J, \& Shenkar, O. (1996). Handbook for international management research. Cambridge: Blackwell.

Raghuram, P, \& Madge, C. (2006). Towards a method for postcolonial development geography? Possibilities and changes. Singapore Journal of Tropical Geography, 27(3), 270288.

Redding, S. G. (1994). The comparative management theory zoo: getting the elephants and ostriches and even dinossaurs from the jungle into the iron cages. In B. Toyne \& D. Nigh (Eds.). International business inquiry: an emerging vision (pp. 416-439). Columbia: University of South Carolina Press.

Redding, S. G. (2005). The thick description and comparison of societal systems of capitalism. Journal of International Business Studies, 36(2), 123-155.

Reingold, N, \& Rothenberg, M. (Eds.). (1987). Scientific colonialism: a cross-cultural comparison. Washington DC: Smithsonian Institution Press.

Roberts, K. H. (1970). On looking at an elephant: an evaluation of cross-cultural research related to organizations. Psychological Bulletin, 74(5), 327-350.

Said, E. (1993). Culture and imperialism. London: Vintage.

Said, E. W. (1978). Orientalism: western conceptions of the orient. New York: Pantheon Books.

Santos, B. S. (2007). Para além do pensamento abissal: das linhas globais a uma ecologia dos saberes. Revista Crítica de Ciências Sociais, 78, 3-46.
Schollhammer, H. (1969). The comparative management theory jungle. Academy of Management Journal, 12(1), 81-97.

Schollhammer, H. (1973). Strategies and methodologies in international business and comparative management research. Management International Review, 13(6), 17-32.

Schollhammer, H. (1975). Current research on international and comparative management issues. Management International Review, 15(2-3), 29-45.

Scholte, J. A. (2000). Globalization: a critical introduction. Basingstoke: Palgrave.

Smith, L. T. (1999). Decolonizing methodologies: research and indigenous peoples. London: Zed Books and University of Otago Press.

Springhall, J. (2001). Decolonization since 1945: the collapse of European overseas empires. Basingstoke: Palgrave Macmillan.

Thomas, N. (1994). Colonialism's culture: anthropology, travel and government. Cambridge: Polity Press.

Thrift, N. (2005). Knowing capitalism. London: Sage.

Tsui, A. S. (2007). From homogenization to pluralism: international management research in the academy and beyond. Academy of Management Journal, 50(6), 1353-1364.

Tucker, V. (1999). The myth of development. In R. Munck \& D. O'Hearn (Eds.). Critical development theory (pp. 1-26). London: Zed Books.

Weinshall, T. D. (1977). Culture and management. London: Penguin.

Westwood, R. I. (2001). Appropriating the other in the discourse of comparative management. In R. I. Westwood \& S. Listead (Eds.). The language of organization (pp. 241-262). London: Sage Publications.

Westwood, R. I. (2004). Towards a postcolonial research paradigm in international business and comparative management. In R. Marschan-Piekkari \& C. Welch (Eds.). Handbook of qualitative research methods for international business (pp. 56-83). Chentenham: Edward Elgar.

Westwood, R. I, \& Jack, G. (2007). Manifesto for a post-colonial international business and management studies: a provocation. Critical Perspectives on International Business, 3(3), 246-265. 